\title{
Probing canonical geometrical objects by digital spiral imaging
}

\author{
Gabriel Molina-Terriza \\ gabriel.molina@icfo.es
}

Liis Rebane

Juan P. Torres

Lluis Torner

Silvia Carrasco
ICFO - Institut de Ciencies Fotoniques, and Universitat Politecnica de Catalunya, Mediterranean Technology Park, 08860 Castelldefels (Barcelona), Spain

ICFO - Institut de Ciencies Fotoniques, and Universitat Politecnica de Catalunya, Mediterranean Technology Park, 08860 Castelldefels (Barcelona), Spain

ICFO - Institut de Ciencies Fotoniques, and Universitat Politecnica de Catalunya, Mediterranean Technology Park, 08860 Castelldefels (Barcelona), Spain

ICFO - Institut de Ciencies Fotoniques, and Universitat Politecnica de Catalunya, Mediterranean Technology Park, 08860 Castelldefels (Barcelona), Spain

Department of Chemistry and Chemical Biology, Harvard University, Cambridge, MA 02138

We recently proposed a novel concept to remotely acquire information of objects, based on the discrete orbital angular momentum of light. Here we use two different schemes for implementing the technique. In order to test the two acquisition methods and to compare their results, we use as target the simplest canonical phase jump. [DOI: 10.2971/jeos.2007.07014]

Keywords: Imaging

\section{INTRODUCTION}

A major application of optics is remote sensing and probing of a variety of properties of matter. Many techniques based on most known properties of light have been elucidated over the years to achieve that goal. Available techniques rely, e.g., on a particular use of the energy spectra of light signals, their longitudinal and transverse linear momenta, their temporal and spatial coherence, and their angular momentum associated with the polarization of the electromagnetic fields. Nevertheless, the angular momentum can contain not only a spin contribution associated to the polarization, but also an orbital contribution associated to the spatial profile of the light beam amplitude and phase-front [1]. Within the paraxial regime, both contributions can be measured and manipulated separately [2]. The orbital contribution is finding important applications in a variety of areas, that range from optical tweezing in biosciences, to microfluidics, micromechanics, and quantum information (see, e.g., [3]-[22], and references therein).

While the spin angular momentum is a workhorse in remote sensing across the electromagnetic spectrum, the potential of the orbital angular momentum has not been fully exploited. The former, often termed polarimetry, is based on the information codified in a two-dimensional space, while the latter is based on the information encoded in the infinite-dimensional spiral spectrum. We recently put forward the idea of using such discrete spiral spectra to remotely acquire information of suitable objects [23]. The technique comprises illuminating the target with a light beam with a convenient spatial shape, amplitude and phase, expanding the reflected or transmitted signal into spiral eigenstates of orbital angular momentum, and acquiring information of the target by analyzing the corresponding spiral spectrum. The shape of the spiral spectrum, the associated spiral bandwidth, or the weights of prescribed eigenstates carry the sought-after information. The concept should be suited for particular physical settings specially sensitive to the spiral phase structure of the probing signal, and in applications where a multidimensional codification scheme may be beneficial.

In this paper, we present a proof-of-principle implementation of the technique based on two different acquisition schemes. We present their characteristics, and discuss their main advantages and drawbacks. The experiments are conducted with a simple canonical object to elucidate the intrinsic potential of the schemes. The simplicity of the object allows us to test the quantitative results of the schemes and to compare them.

\section{CONCEPT}

A paraxial light beam propagating in the $z$ direction, $F(\rho, \phi, z)$, can be decomposed into spiral modes as

$$
F(\rho, \phi, z)=\sum_{m} A_{m}(\rho, z) \exp (i m \phi) / \sqrt{2 \pi}
$$

where

$$
A_{m}(\rho, z)=\frac{1}{\sqrt{2 \pi}} \int_{0}^{2 \pi} d \phi F(\rho, \phi, z) \exp (-i m \phi),
$$

and $(\rho, \phi)$ are the cylindrical coordinates in the transverse plane. The amplitude of the spiral modes has an azimuthal 
dependence of the form $\exp (\operatorname{im} \varphi)$, so that the phase of each mode is twisted around the center of the beam $m$ times. The index $m$ is usually called the winding number, or topological charge of the phase singularity located at $\rho=0$ of the corresponding mode.

The terms $A_{m}(\rho, z)$ describe the radial dependence associated with every spiral mode. The OAM content of the light beam is determined by the distribution $\left\{C_{m}\right\}$, where $C_{m}=$ $\int_{0}^{\infty} d \rho \rho\left|A_{m}(\rho, z)\right|^{2}$ [21]. Importantly, the value of $C_{m}$ is conserved through free-space propagation [24], i.e., it does not depend on the coordinate $z$. The total orbital angular momentum of the beam writes $\left\langle L_{z}\right\rangle \propto \sum_{m} m C_{m}$. As $m$ can be either positive or negative, the knowledge of $C_{m}$ can provide useful information even for beams with a zero total orbital angular momentum.

In the kind of applications we are considering, $F$ is the result of applying a well characterized input beam to an unknown target. In the simplest case, one can write $F(\rho, \phi)=$ $G(\rho, \phi) T(\rho, \phi)$, where $G$ is the probe beam, typically a Gaussian beam, and $T$ is the complex transmissivity (or reflectivity) of the object under investigation.

The goal is to evaluate the magnitude of a specific characteristic of the target, from the measurement of the total, or partial, OAM content of the light beam that is reflected, or transmitted. There are several techniques proposed to retrieve experimentally the OAM content of a light beam [25]- [27]. Each one of them exhibits advantages and drawbacks, with a varying degree of difficulty for its practical implementation.

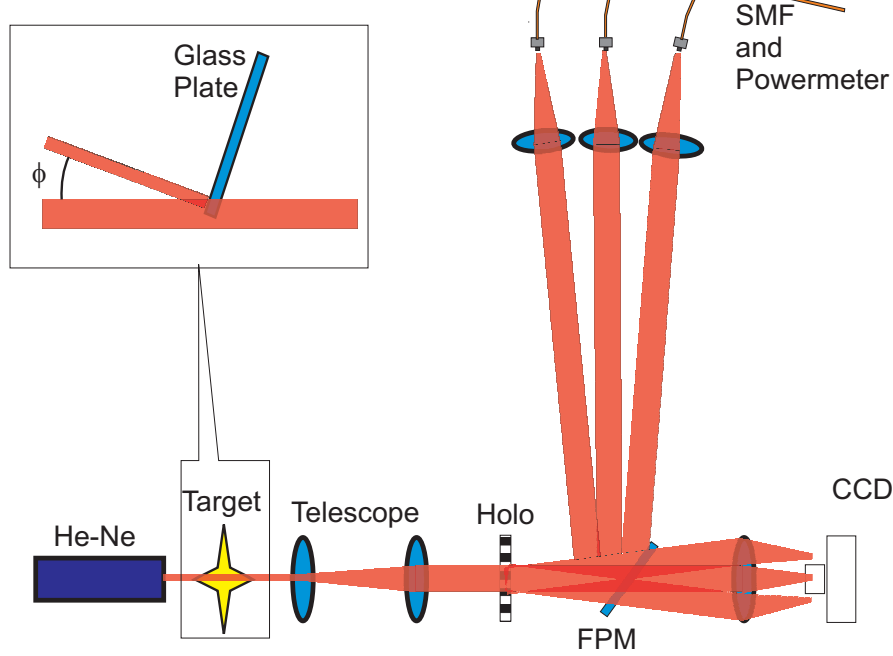

FIG. 1 Experimental Set-up. A He-Ne laser illuminates the target. The resulting beam is expanded with a telescope to take advantage of the whole pattern of a binary fork hologram (Holo), which diffracts the incoming light into several different orders, each one with an additional azimuthal phase. Finally with a flip-mount mirror (FPM), one can direct light into either of the two different measuring systems: Coupling the whole diffraction order into a single-mode fiber (SMF), or Fourier transforming it with a lens and measuring the result with a CCD camera. In the inset one can see a close-up of the target, which in our case consists of a simple glass plate which delays part of the beam. The delay can be controlled with the tilting angle of the glass plate $(\Phi)$.

We consider here two quantities closely related to the sought- after amplitude of the spiral modes, which can be readily extracted experimentally. In order to measure both quantities, we implement the experimental arrangement depicted in Figure 1. Light is directed to either detection scheme with a flipmount mirror, so that one can perform a proper comparison between both detection schemes.

One of the measuring systems is composed of a fork-like holographic mask [28, 29], followed by a single mode fiber (SMF) coupler. The computer generated hologram (CGH) splits the incoming beam into several orders of diffraction, each one of them travelling in an slightly different direction. For each outgoing diffraction order, the CGH impinges a different azimuthal phase dislocations to the incoming light field.

The whole beam from the desired diffraction order is then coupled into the SMF, which acts as a projector onto the corresponding Gaussian mode of the monomode fiber (beam width $w_{f}$ ). The detected power is thus given by (see Appendix A)

$$
N_{m}=\frac{4}{w_{f}^{2}}\left|\int_{0}^{\infty} d \rho \rho A_{m}(\rho) \exp \left(-\rho^{2} / w_{f}^{2}\right)\right|^{2},
$$

The coupling of the diffraction order $m$ into the single mode fiber is thus a measurement on the corresponding $m$ spiral mode, as expressed in Eq. (3).

To date, such a technique to retrieve partial information about the spiral spectrum has been mainly used in quantum information experiments $[30,31]$. Notice that this detection scheme provides information about the overlap of each of the modes $A_{m}(\rho)$ with a Gaussian beam, not with the Laguerre-Gaussian basis. The method is particularly interesting when the light used to probe the target has also a Gaussian shape.

The second system implemented also makes use of a CGH, but this time the CGH is followed by a CCD camera that records each of the diffracted orders coming out from the hologram, after the application of a Fourier transform with a lens. From each image taken, we are only interested in the value of the pixel which is at the central point of the corresponding diffraction order. As shown in the Appendix A, this quantity can be expressed as

$$
M_{m}=2 \pi\left|\int_{0}^{\infty} d \rho \rho A_{m}(\rho)\right|^{2} .
$$

This scheme provides information about the average value of the radial mode. Generally speaking, this method provides different information about the spiral spectrum than Eq. (3), as it is readily visible in both formulas.

Note that the presence of light at the central spot of the mode has been previously used by several authors, e.g., by direct eye-inspection, to monitor qualitatively the presence of the corresponding mode $[32,33]$. In sharp contrast, it must be properly appreciated that in our method such measurement is used to obtain a quantitative measure of the weight of the mode.

\section{SET-UP}

In our experiments we use a $10 \mathrm{~mW}$ He:Ne laser with a TEM 00 mode as the probe beam. The laser beam is directed onto the 
target. The target is a canonical phase jump. The phase step is produced with a thin microscope glass plate, which can be moved in and out of the laser beam with a stepper motor. We vary the inclination of the plate, to introduce different phase delays.

The effect of the target on the probe beam writes,

$$
T\left(\rho, \phi ; x_{0}\right)= \begin{cases}t \exp (\text { i } \gamma) & \text { if } \rho \cos (\phi)>x_{0} \\ 1 & \text { if } \rho \cos (\phi) \leq x_{0}\end{cases}
$$

where $T\left(\rho, \phi ; x_{0}\right)$ is the transmittance of the target, $t$ is the modulus of the transmittance of the plate, $\gamma$ is the phase jump produced by the plate, and $x_{0}$ is the location of the edge of the plate. We measure the angle of the plate using the reflected beam from the input face of the glass.

The CGH we use for both measuring systems is a binary amplitude hologram, which has enough efficiency for several orders of diffraction. In order to avoid inaccuracies due to the binary nature of the hologram, we use a $4 x$ telescope to expand the incoming field that illuminates it.

The output field from the target is analyzed with the two measurement devices described above. In the first measuring system, we place the fiber coupler on a translation stage. With the help of a mirror and displacing the fiber coupler with a translation stage, we couple every different order of diffraction into the fiber. The fiber coupler consists on two lenses which can be moved independently. This system allows the coupling of more than $80 \%$ of the power of a $\mathrm{TEM}_{00}$ mode into the single mode fiber.

The second analyzing method replaces the whole fiber coupler by a CCD camera which is placed in the focal plane of a lens. Each frame taken by the camera can store several orders of diffraction simultaneously, which can be analyzed independently. The central pixel of every diffraction order recorded in the camera, contains the information of the quantity $M_{m}$, as given by Eq. (4). A simple numerical analysis extracts the information contained in the central pixel value from a series of images stored in the CCD camera. (a)

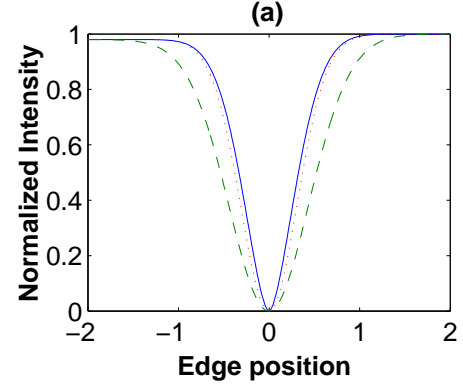

(b)

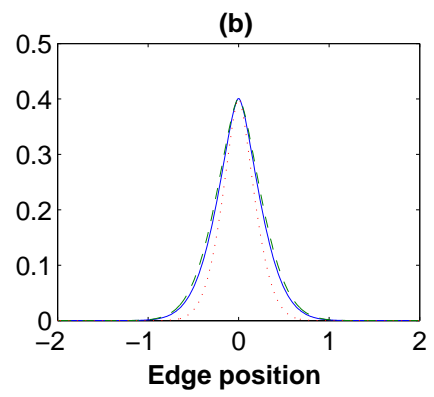

FIG. 2 Numerically calculated values of $C_{m} / C_{0}(\infty)$ (continuous line), $M_{m} / M_{0}(\infty)$ (dashed line) and $N_{m} / N_{0}(\infty)$ (dotted line) when moving the edge of the glass plate (see text for details). The edge position is normalized to the beam width. (a) $m=0$, (b) $m=1$.

Figure 2 shows how the magnitudes $N_{m}$ and $M_{m}$ are related to the sought-after weight of the spiral modes $C_{m}$ using model in
Eq. (5) for the glass plate. More specifically, it plots the weight of the spiral modes $m=0$ and $m=1$ as a function of the position of the edge of the slide $\left(x_{0}\right)$, compared with $N_{m}$ and $M_{m}$. Figure 2 shows that $N_{0,1}$ and $M_{0,1}$ are good estimates of the weight of the corresponding modes $m=0,1$ in our particular experimental configuration.

\section{EXPERIMENTAL RESULTS}

In Figure 3 we show the results of our experiment for a particular inclination of the glass plate. In all figures the horizontal axis represents the displacement of the edge of the glass-plate. The two curves in Figure 3a compare the results obtained for a given inclination of the glass plate (producing a phase jump of $\gamma \approx \pi$ ) with the two methods: with the CCD camera (circles) and with the fiber coupler (crosses). The data represents, respectively, $M_{0}\left(x_{0}\right)$ and $N_{0}\left(x_{0}\right)$.

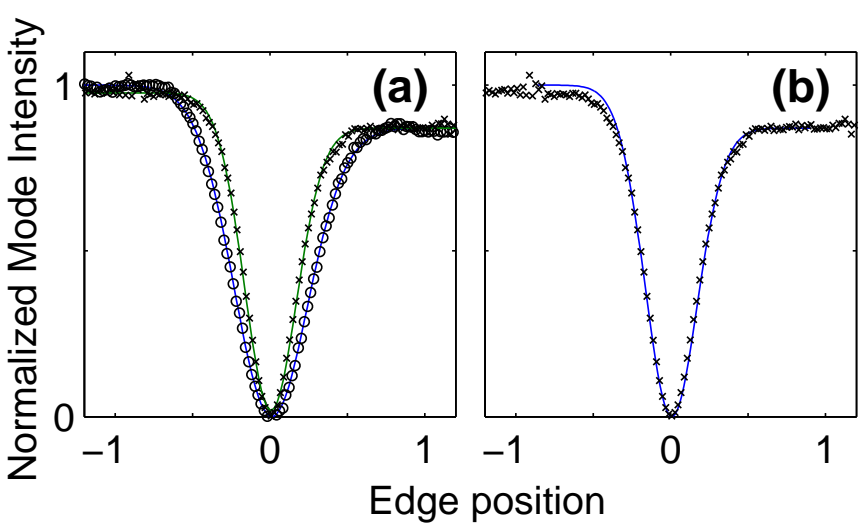

FIG. 3 Comparison of mode amplitudes using the two implemented DSI set-ups with a typical setting of the glass plate and measuring in the mode $m=0$. Horizontal axis: edge position with respect to the centre of the beam. a) CCD method (circles) and fiber method (crosses). Solid lines, best fit using the theoretical model (details in text). b) Fiber method (crosses). Solid line is best fit of Figure za for the CCD method, rescaled in the horizontal axis by a factor of $\sqrt{2}$ (details in text).

As shown in the Appendix A, for this particular scheme, the two measured quantities, as given by Eqns. (3) and (4), verify

$$
N_{m}\left(x_{0}\right) \propto M_{m}\left(x_{0} \frac{w_{G}}{\sqrt{w_{G}^{2}+w_{f}^{2}}}\right) .
$$

If the coupling of the probe Gaussian beam into the fiber is optimal, then $w_{G}=w_{f}$ and the above relation simplifies to $N_{m}\left(x_{0}\right) \propto M_{m}\left(x_{0} / \sqrt{2}\right)$, which applies to the results shown in Figure 3 . The same functions can be used to obtain the best fits of both the CCD method and the fiber coupler one. The solid lines in Figure 3 represent the best fits to the data, modeling the glass plate with Eq. (5). The free parameters used are the value of the phase jump, the attenuation of the glass plate, the offset of the stepper motors, the intensity of the light beam and the width (which in the case of $M_{m}$ corresponds to the beam width and in the case of $N_{m}$ to $w_{G} / \sqrt{w_{G}^{2}+w_{f}^{2}}$ ).

In Figure $3 b$ one can observe the similarity of the results obtained with both methods. In this figure, we plot the data from 
the measurement with the fiber coupler (crosses). The solid line is the best fit for the CCD camera data from Figure 3a, just re-scaled by a factor $\sqrt{2}$, showing that Eq. (6) applies well to our experimental data. Similar results were found for all values of the phase jumps. In Figure 4 we show a few of the complete snapshots taken with the CCD camera. Notice that although the whole intensity pattern of the transmitted beam can be rather complex, as shown in Figure 4, the intensity of the central point of the image, follows Eq. (4).
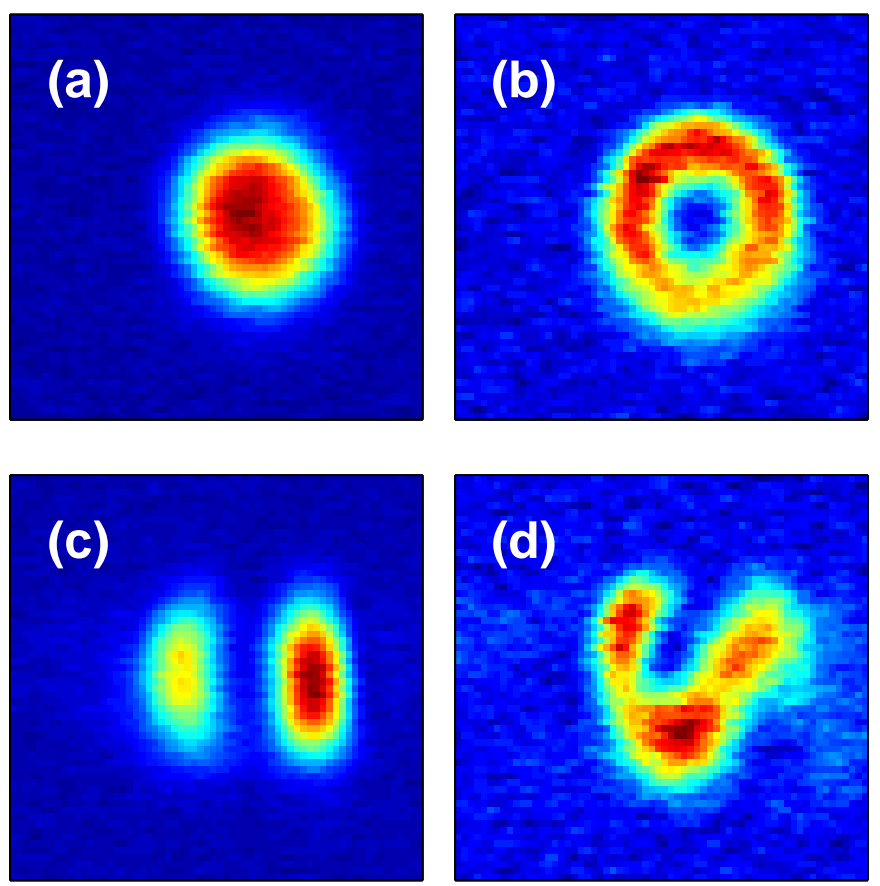

FIG. 4 Typical images recorded with the analyzing system. First row, edge of the glass plate is completely out of the beam. Second row, edge of the glass plate is located in the middle of the probe beam $\left(x_{0}=0\right)$. First column: mode $m=0$, second column: mode $m=1$. We note that the beam in (b) shows a slight asymmetry due to a small misalignment of the hologram position. In (c), the left lobe is covered by the glass plate and due to the reflectivity of the glass, has a lower power than the right lobe.

The central result of our experiments is presented in Figure 5, which depicts the different curves obtained by changing the inclination of the glass plate. Each curve is labeled with the phase jump obtained from a best fit using the models explained above. The two figures show the results for two different modes using the CCD camera method. The quality of the fits confirm the suitability of the model and shows how different phase jumps were effectively detected with the DSI method.

It is worth pointing out additional features which highlight the potential of the DSI analysis. In Figure 5a, one can observe that the transmission of the glass plate changes with its inclination. We checked that this change in the transmission oscillates with the inclination of the plate. Due to the fact that the reflectivity of the two faces of the plate is finite, a Fabry-Perot cavity is formed inside the cavity, which explains the changes in the transmitted power of the plate.

Another interesting effect can be seen in Figure 5b, where we plot the weight of the mode $m=1$. When the beam is well inside the glass plate, ideally the contribution of the modes

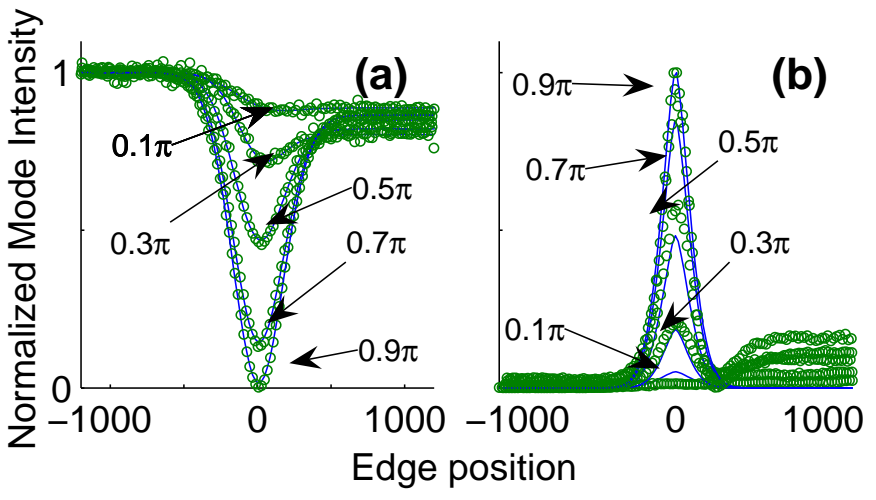

FIG. 5 Amplitude of modes for different glass plate inclinations. The horizontal axis is the position of the glass plate edge, with respect to the beam centre. Circles: experimental data, solid lines: best fit. Each curve produced with a different inclination of the glass plate, labels indicate the phase jump given by the best fit. a) Mode $m=0$. Normalized by the intensity of the laser, retrieved from the best fit. b) Mode $m=1$. All curves normalized to the maximum of the curve $\gamma=0.9 \pi$.

$m \neq 0$ should be zero. This is due to the fact that the probe beam is Gaussian and the glass plate maintains the symmetry of the incoming beam, since it only introduces a uniform phase and amplitude modulation. Nevertheless, one can observe that the curves in Figure $5 b$ show a small but nonvanishing contribution of the mode $m=1$. The most likely source of this deviation is a small displacement of the probe beam, due to the refraction of the light beam in the glass plate. Such a deviation might be difficult to observe with other methods, since the displacement is usually much smaller than the width of the beam. The use of DSI can thus make possible to conduct ultra precise measurements that might be difficult to achieve by other means [34]. In this context, note that the proper use of a multimodal expansion of a displaced Gaussian beam has recently afforded sub-shot-noise measurements of small beam displacement [35].

\section{CONCLUDING REMARKS}

We have presented a proof-of-principle experimental implementation of a DSI scheme using the simplest canonical target. We have tested and compared two different methods for experimentally acquiring the spiral spectrum of a light beam. The target of our experiments was a phase jump, which was used to elucidate the accuracy and robustness of the detection schemes. Under suitable conditions, the two schemes provide the same information about the target.

Our observations confirm that DSI can remotely obtain geometrical information about amplitude and phase objects, an ability that might be used to complement information provided by other methods, or to probe and detect targets difficult to spot by standard methods. In particular notice that specialized surface metrology and orientational sorting (e.g., $[23,36,37])$, or edge-contrast enhancement of simple targets, are direct possibilities worth investigating.

While the high sensitivity to phase and amplitude variations of the scheme advanced here, might pose restrictions on its practical applicability in turbid or in disordered media, it 
might likewise be used to gain information about the random properties of the corresponding light path. Finally, notice that the concept can also be extended to the digital spiral spectra of near fields, and thus to probing targets at the nanoscale.

\section{A APPENDIX}

In this appendix we derive the main equations used throughout the paper. We start with Eq. (1), the decomposition into spiral modes of the field being reflected or transmitted by the target. We repeat here the spiral decomposition for the sake of clarity:

$$
\begin{array}{r}
F(\rho, \phi, z)=\sum_{n} A_{n}(\rho, z) \exp (i n \phi) / \sqrt{2 \pi} \\
A_{n}(\rho, z)=\frac{1}{\sqrt{2 \pi}} \int_{0}^{2 \pi} d \phi F(\rho, \phi, z) \exp (-i n \phi) .
\end{array}
$$

When the field $F(\rho, \phi, z)$, is diffracted by an hologram placed at $z_{0}$, each diffraction order acquires a different azimuthal phase. The complex amplitude $E_{m}=F\left(\rho, \phi, z_{0}\right) \exp (-i m \phi)$ of the diffraction order $m$ can be written as

$$
\left.E_{m}(\rho, \phi)=\sum_{n} A_{n}\left(\rho, z_{0}\right) \exp [i(n-m) \phi]\right) / \sqrt{2 \pi} .
$$

After selecting this diffraction order, the output face of the hologram is imaged onto a monomode fiber. For a monomode fiber long enough, the beam which exits the fiber has a spatial shape which matches the fundamental mode of the fiber. This mode can be approximated by a Gaussian mode with width $\tilde{w}_{F}$. Thus the optical power measured after the fiber is

$$
N_{m}=\frac{2}{\pi{\tilde{w_{F}}}^{2}}\left|\int_{0}^{2 \pi} d \phi \int_{0}^{\infty} d \rho \rho E_{m}(\kappa \rho, \phi) \exp \left(-\rho^{2} / \tilde{w}_{F}^{2}\right)\right|^{2}
$$

with $\kappa$ being the magnification factor of the imaging system. After performing the azimuthal integral, the only remaining term of the sum (A.1) is $n=m$ and the expression for $N_{m}$ gives

$$
N_{m}=\frac{4}{{\tilde{w_{F}}}^{2}}\left|\int_{0}^{\infty} d \rho \rho A_{m}(\kappa \rho) \exp \left(-\rho^{2} / \tilde{w}_{F}^{2}\right)\right|^{2},
$$

which is the same result presented above in Eq. (3), when we make use of $w_{F}=\kappa \tilde{w}_{F}$. This is the width of the mode of the fiber after traversing the imaging system backwards onto the hologram.

For the derivation of $M_{m}$, we select an order of diffraction from the hologram, and perform a Fourier transform operation. The field $\mathcal{E}_{m}\left(\rho_{k}, \phi_{k}\right)$ reads

$$
\begin{aligned}
\mathcal{E}_{m}\left(\rho_{k}, \phi_{k}\right) & =\sum_{n} \frac{1}{\sqrt{2 \pi}} \int_{0}^{\infty} \rho d \rho \times \\
& \int_{0}^{2 \pi} d \phi A_{n}(\rho) \exp [i(n-m) \phi] \exp \left[i \rho_{k} \rho \cos \left(\phi-\phi_{k}\right)\right]
\end{aligned}
$$

After performing the integration into the azimuthal coordinate, one obtains

$$
\begin{gathered}
\mathcal{E}_{m}\left(\rho_{k}, \phi_{k}\right)=\sum_{n} \frac{\sqrt{2 \pi}}{i^{(n-m)}} \exp \left[i(n-m) \phi_{k}\right] \\
\int_{0}^{\infty} \rho d \rho A_{n}(\rho) J_{(n-m)}\left(\rho_{k} \rho\right) .
\end{gathered}
$$

The only Bessel function with a nonzero value in the origin is $J_{0}$. Therefore, $\mathcal{E}_{m}\left(\rho_{k}=0\right)=\frac{\sqrt{2 \pi}}{i^{n}} \int_{0}^{\infty} \rho d \rho A_{m}(\rho) J_{0}(0)$ and one readily finds that $M_{m}=\left|\mathcal{E}_{m}\left(\rho_{k}=0\right)\right|^{2}$, which is the soughtafter expression.

Finally, we address the particular case where the two intrinsically different measurements from Eqs. (3 and 4) provide the same information. For the case that the probe beam is gaussian, the radial dependence of the amplitude of the spiral modes write $A_{m}(\rho)=C a_{m}(\rho) \exp \left(-\rho^{2} / w_{G}^{2}\right)$, with $C$ and $w_{G}$ being the amplitude and width of the gaussian probe beam, and $a_{m}(\rho)$ the spiral information due to the target. The quantities $N_{m}$ and $M_{m}$ now read

$$
\begin{aligned}
& N_{m}=\frac{4}{w_{f}^{2}}\left|C \int_{0}^{\infty} d \rho \rho a_{m}(\rho) \exp \left(-\rho^{2} / w_{G}^{2}\right) \exp \left(-\rho^{2} / w_{f}^{2}\right)\right|^{2} \\
& M_{m}=2 \pi\left|C \int_{0}^{\infty} d \rho \rho a_{m}(\rho) \exp \left(-\rho^{2} / w_{G}^{2}\right)\right|^{2}
\end{aligned}
$$

The expressions of $N_{m}$ and $M_{m}$ are very similar, although the Gaussian modes in the overlap integral have different widths. Nevertheless, when the set of spiral modes characterizing the target depend on a free parameter $x_{0}$ in the following way $a_{m}\left(\rho / x_{0}\right)$, then the two measures follow the relation $N_{m}\left(x_{0}\right) \propto$ $M_{m}\left(x_{0} w_{G} / \sqrt{w_{G}^{2}+w_{f}^{2}}\right)$ and provide the same information.

This is exactly the case for the canonical phase jump used in the experiments, where the $x_{0}$ free parameter is the displacement of the phase jump with respect to the center of the beam.

\section{ACKNOWLEDGEMENTS}

This work has been partially supported by the Government of Spain through grants TEC2005-07815 and FIS2004-03556. It has also been partially supported by the European Commission under the Integrated Project Qubit Applications (QAP) funded by the IST directorate as Contract No. 015848. Gabriel Molina-Terriza acknowledges support by the Ramon-y-Cajal and ICREA Junior program.

\section{References}

[1] L. Allen, S. M. Barnett, and M. J. Padgett, “Optical angular momentum" (Institute of Physics Publishing, 2003).

[2] A. T. O’Neil, I. MacVicar, L. Allen, and M. J. Padgett, "Intrinsic and extrinsic nature of the orbital angular momentum of a light beam" Phys. Rev. Lett. 88, 053601 (2002).

[3] H. He, M. E. J. Friese, N. R. Heckenberg, and H. RubinszteinDunlop, "Direct observation of transfer of angular momentum to absorbing particles from a laser beam with a phase singularity" Phys. Rev. Lett. 75, 826-829 (1995).

[4] N. R. Heckenberg, T. A. Nieminen, M. E. J. Friese, and H. Rubinsztein-Dunlop, "Trapping microscopic particles with singular beams" in International Conference on singular Optics, M. S. Soskin ed., Proc. SPIE 3487, 46-53 (1998).

[5] S. J. Parkin, T. A. Nieminen, N. R. Heckenberg, and H. RubinszteinDunlop, "Optical measurement of torque exerted on an elongated object by a noncircular laser beam" Phys. Rev. A 70, 023816 (2004). 
[6] N. B. Simpson, K. Dholakia, L. Allen, and M. Padgett, "Mechanical equivalence of spin and orbital angular momentum of light: An optical spanner" Opt. Lett. 22, 52-54 (1997).

[7] L. Paterson, M. P. MacDonald, J. Arlt, W. Sibbett, P. E. Bryant, and K. Dholakia, "Controlled rotation of optically trapped microscopic particles" Science 292, 912-914 (2001).

[8] K. Dholakia, G. Spalding, and M. MacDonald, "Optical tweezers: the next generation" Phys. World 15, 31 (2002).

[9] E. Santamato, A. Sasso, B. Piccirillo, and A. Vella, "Optical angular momentum transfer to transparent isotropic particles using laser beam carrying zero average angular momentum" Opt. Express 10, 871-878 (2002). http://www.opticsexpress.org/abstract.cfm?URI=0PEX-10-17-871

[10] E. Santamato, "Photon orbital angular momentum: Problems and perspectives" Fortschr. Phys. 52, 1141-1153 (2004).

[11] P. Galajda and P. Ormos, "Complex micromachines produced and driven by light" Appl. Phys. Lett. 78, 249-251 (2001).

[12] K. Ladavac and D. G. Grier, "Microoptomechanical pumps assembled and driven by holographic optical vortex arrays" Opt. Express 12, 1144 (2004).

[13] D. G. Grier, "A revolution in optical manipulation" Nature (London) 424, 810-816 (2003).

[14] A. Jesacher, S. Fürhapter, S. Bernet, and M. Ritsch-Marte, "Shadow effects in spiral phase contrast microscopy" Phys. Rev. Lett. 94, 233902 (2005).

[15] G. Swartzlander, "Peering into darkness with a vortex spatial filter" Opt. Lett. 26, 497-499 (2001).

[16] D. Mawet, P. Riaud, 0. Absil, and J. Surdej, "Annular groove phase mask coronagraph" Astrophys. J. 633, 1191-1200 (2005).

[17] E. G. Johnson, J. Stack, and Ch. Koehler, “Light coupling by a vortex lens into graded index fiber" J. Lightwave Technol. 19, 753-758 (2001).

[18] R. J. Voogd, M. Singh, S. Pereira, A. van de Nes, and J. Braat, "The use of orbital angular momentum of light beams for super-high density optical data storage" OSA Annual Meeting, Rochester, NY, October 2004, paper FTuG14.

[19] H. H. Arnaut and G. A. Barbosa, "Orbital and intrinsic angular momentum of single photons and entangled pairs of photons generated by parametric down-conversion" Phys. Rev. Lett. 85, 286-290 (2000).

[20] A. Mair, A. Vaziri, G. Weihs, and A. Zeilinger, "Entanglement of the orbital angular momentum states of photons" Nature (London) 412, 313-316 (2001).

[21] G. Molina-Terriza, J. P. Torres, and L. Torner, "Management of the angular momentum of light: preparation of photons in multidimensional vector states of angular momentum" Phys. Rev. Lett. 88, 013601 (2002).

[22] G. Molina-Terriza, A. Vaziri, R. Ursin, and A. Zeilinger, "Experimental quantum coin tossing" Phys. Rev. Lett. 94, 040501 (2005).
[23] L. Torner, J. P. Torres, and S. Carrasco, "Digital spiral imaging," Opt. Express 13, 873-881 (2005). http://www.opticsinfobase.org/abstract.cfm?URI=0e-13-3-873

[24] S. J. Enk and G. Nienhuis. "Eigenfunction description of laser beams and orbital angular momentum of light" Opt. Commun. 94, 147 (1992).

[25] J. Leach, M. J. Padgett, S. M. Barnett, S. Franke-Arnold, and J. Courtial, "Measuring the orbital angular momentum of a single photon" Phys. Rev. Lett. 88, 257-901 (2002).

[26] M. V. Vasnetsov, J. P. Torres, D. V. Petrov, and L. Torner, “Observation of the orbital angular momentum spectrum of a light beam" Opt. Lett. 28 2285-2287 (2003).

[27] R. Zambrini and S. M. Barnett, "Quasi-intrinsic angular momentum and the measurement of its spectrum" Phys. Rev. Lett. 96, 113901 (2006).

[28] V. Y. Bazhenov, M. V. Vasnetsov, and M. S. Soskin, "Laser beams with screw dislocations in their wavefront" JETP Lett. 52, 429-431 (1990).

[29] N. R. Heckenberg, R. McDuff, C. P. Smith, H. Rubinsztein-Dunlop, and M. J. Wegener "Laser beams with phase singularities" Opt. Quant. Electron. 24, S951-S962 (1999).

[30] Alipasha Vaziri et al, "Superpositions of the orbital angular momentum for applications in quantum experiments" J. Opt. B: Quantum S. 0. 4 S47-S51, (2002).

[31] G. Molina-Terriza, A. Vaziri, J. Rehacek, Z. Hradil, and A. Zeilinger, “Triggered Qutrits for Quantum Communication Protocols" Phys. Rev. Lett. 92, 167903 (2004).

[32] P. Di Trapani, A. Beržanskis, S. Minardi, S. Sapone, and W. Chinaglia, "Observation of optical vortices and $J_{0}$ Bessel-like beams in quantum-noise parametric amplification" Phys. Rev. Lett. 81, 5133-5136 (1998).

[33] G. Cibson, J. Courtial, M. Padgett, M. Vasnetsov, V. Pas'ko, S. Barnett, and S. Franke-Arnold, "Free-space information transfer using light beams carrying orbital angular momentum" 0pt. Express 12, 5448-5456 (2004). http://www.opticsinfobase.org/abstract.cfm?URI=0e-12-22-5448

[34] R. Dasgupta, and P. K. Gupta, "Experimental observation of spinindependent transverse shift of the centre of gravity of a reflected Laguerre-Gaussian light beam" 0pt. Comm. 257, 91 (2006).

[35] V. Delaubert, N. Treps, C. C. Harb, P. K. Lam, and H. A. Bachor, "Quantum measurements of spatial conjugate variables: displacement and tilt of a Gaussian beam" Opt. Lett. 31, 1537-1539 (2006).

[36] C. Rockstuhl, M. Salt, and H. P. Herzing, "Theoretical and experimental investigation of phase singularities generated by optical micro- and nano-structures" J. Opt. A: Pure App. Opt. 6, S271-S276 (2004).

[37] A. V. Failla, H. Qian, H. Qian, A. Hartschuh, and A. J. Meixner, "Orientational imaging of subwavelength Au particles with higher order laser modes" Nano Lett. 6, 1374-1378 (2006). 